\title{
On the Shielding Effectiveness Calculation of Enclosures Through Measurements in Reverberation Chambers
}

\author{
Angelo Gifuni, IEEE Member, Luca Bastianelli, IEEE Student Member, Gabriele Gradoni, IEEE Member, Franco Moglie, \\ IEEE Senior Member, Stefano Perna, IEEE Senior Member, Christopher Smartt, and Valter Mariani Primiani, IEEE Senior \\ Member.
}

\begin{abstract}
The IEC 61000-4-21 and IEEE 299.1 documents report similar but not equal procedures to measure the shielding effectiveness (SE) of enclosures through the use of Reverberation Chambers (RCs). In this paper, it is shown that these two measurement procedures can be merged in a single, enhanced, one. In particular, it is shown that the evaluation of the SE of enclosure in Annex H of IEC 61000-4-21, which exploits only mechanical stirring, can be improved by using the hybrid stirring, which includes also the frequency stirring thus increasing the number of uncorrelated samples. On the other hand, it is shown that at low frequencies the evaluation of the SE of enclosures based on the definition in IEEE 299.1 can be improved through the use of the maximum values of measured powers rather than the average ones. The single, enhanced, proposed procedure provides more prudent $S E$ values than the two standards at the low frequencies, especially for standard IEEE 299.1. The presented measurement approach is supported by results obtained in three different laboratories.
\end{abstract}

Index Terms - Nested reverberation chambers, enclosures, shielding effectiveness calculation.

\section{INTRODUCTION}

Reverberation Chamber (RC) is an electrically large metallic cavity where the internal field is properly randomized by a Frequency electronic stirring (FS) process [1]-[7] and/or a mechanical stirring (MS) including vibrating stirring and position stirring [3], [5]-[7]. In order to make the stirring process more effective, a combination of different stirring processes is used [6]-[9]; however a combination of FS and MS performed through metallic stirrer(s) only is the most common. Combination of stirring techniques, i.e., hybrid stirring (HS), is very important for RCs as it increases the number of uncorrelated samples thus reducing the measurement uncertainty (MU) [8]-[9].

$\mathrm{RCs}$ are used to perform emission and immunity tests on electronic equipment [1], [10] and several other applications including shielding measurements [1], absorption of materials and biological bodies [4], antenna measurements [11], and simulation of wireless multipath environments [12].

Manuscript received November 24, 2020.

A. Gifuni and S. Perna are with the Dipartimento di Ingegneria, Università di Napoli Parthenope, Centro Direzionale di Napoli, Napoli 80143, Italy (e-mail: angelo.gifuni@uniparthenope.it; stefano.perna@uniparthenope.ut).

L. Bastianelli, F. Moglie, and V.M. Primiani are with the Dipartimento di Ingegneria dell'Informazione, Università Politecnica delle Marche, 60131 Ancona, Italy (e-mail: f.moglie@univpm.it; 1.bastianelli@pm.univpm.it; v.mariani@univpm.it).
The shielding effectiveness (SE) measurement for enclosures is a standardized procedure [1], [13] which is usually performed through nested reverberation chambers (NRCs), where the test enclosure forms the inner chamber, and the remaining volume inside the $\mathrm{RC}$ forms the outer chamber. The test enclosure is arranged in the working volume of the RC [14]. In particular, two similar standardized measurement procedures are available: one in the IEC 610004-21 document [1, annex $\mathrm{H}]$ and the other one in the IEEE 299.1 document [13, Sec. 7.2]. In both procedures, the SE is achieved through the ratio of two power-based measurements: the power received inside the enclosure and that received in the outer chamber. The power levels are measured by two antennas operating in receiving mode: one is positioned inside the enclosure and the other one is positioned in the outer chamber. A third antennas feeds the system, as shown in [1], [13], [15]-[18]. Alternative procedures operating in time domain exist, which allow the exploitation of only two antennas [19].

Although the main rationale of the two procedures defined in the IEC 61000-4-21 and IEEE 299.1 documents is the same, these two procedures are not equivalent.

In the IEEE 299.1 [13] case, to achieve the SE, the ratio of measured average powers [13, Sec. 7.2] is exploited. An appropriate FS bandwidth (FSB) is used to achieve stable average values. FS is used in both the inner and outer chamber. A wall-mounted monopole antenna is used inside the enclosure and no mechanical stirring (MS) can be thus adopted inside the enclosure. In the outer chamber, when possible, in addition to the FS also the MS can be used to improve the evaluation of SE [13]. Measurements are performed by a vector network analyzer (VNA) so that both FS and correction for impedance mismatch are considered. The latter is necessary for the monopole antenna inside the enclosure.

In the IEC 61000-4-21 [1] case, to achieve the SE, the ratio between the two maximum values of the two measured powers [1, Annex $\mathrm{H}]$ is exploited. Moreover, only MS, operated through metallic stirrer(s) is adopted, both inside the outer chamber and inside the enclosure, and no FS is exploited. The powers are measured by a power meter (PM), and correction for impedance mismatch is not applied. For this reason, it is

G. Gradoni is with the School of Mathematical Sciences and with the George Green Institute for Electromagnetics Research, Department of Electrical and Electronics Engineering, University of Nottingham, Nottingham NG7 2RD, U.K. (e-mail: gabriele.gradoni@nottingham. ac.uk). C. Smartt is with the George Green Institute for Electromagnetics Research, Department of Electrical and Electronics Engineering, University of Nottingham, Nottingham NG7 2RD, U.K. (e-mail: chris.smartt@nottingham. ac.uk). 
recommended either that the two receiving antennas have the same total efficiency (in free space) or that they are the same antenna model. This helps to reduce the error due to the impedance mismatch, and removes the error due to the radiation efficiency. In fact, the antenna impedance mismatch does not change appreciably at higher frequencies when it is located inside an enclosure where at the minimum working frequency at least 60 modes are supported. On the other hand, it is underlined that a significant change of the impedance mismatch could occur at low frequencies where the modal density is low, thus impairing the accuracy of the overall procedure.

The aim of this paper is to analyze and to compare results from the two standard procedures defined in the IEC 61000-421 and IEEE 299.1 documents, to attempt to merge them in a single enhanced procedure.

We show that the IEEE 299.1 procedure in [13] can be improved at the low frequencies by using the maximum values of the measured powers, rather than the average ones. In particular, the use of the ratios between the maximum values takes into account the high levels of the fields associated to the resonances inside the enclosure. In contrast, the use of the ratios between average values kills the high levels of the field. Such resonances occur at the low frequencies where the modal density is low. Therefore, use of the maximum values rather than the average ones improves the evaluation of the SE of enclosures at the low frequencies compared to the definition in IEEE 299.1; i.e., the MU decreases. At high frequencies, where the resonant behavior of the enclosure and the relevant effect on the SE disappears, the SE from average values of the measured powers can be considered as well in order to reduce the MU.

Specifically, it is shown that when the acquired number of uncorrelated samples is sufficiently large, the SE calculation can be improved for frequencies up to several times the frequency $f_{60}$, being $f_{60}$ the frequency where the enclosure is able to support 60 modes.

On the other side, we show that the IEC 61000-4-21 [1] procedure can be improved by the use of HS (which is obtained by the combination of MS and FS) as well as by the possibility of using a wall-mounted monopole antenna inside the enclosure. Such an improvement implies the use of a VNA in the measurement set-up instead of a PM. The use of HS including FS achieves a larger number of uncorrelated samples at low frequencies where the modal density is low. This increases the probability of exciting resonances (modes) inside the enclosure: the corresponding fields can then couple to the receiving antennas. For this reason, use of HS including FS improves the evaluation of the SE of enclosures in standard IEC 61000-4-21. Moreover, the HS also reduces the MU both at the high and low frequencies. In fact, the HS tends to increase the field uniformity in the enclosure at low frequency, also when the MS is applied only inside the RC.

Summing up, the advantages of HS including the FS, the possible (but not necessary) use of a wall-mounted monopole inside the enclosure, and the calculation of the SE by the ratios between the maximum (rather than average) values of the measured powers, can be merged in a single enhanced measurement procedure, which improves and includes both the standard measurement procedures shown in the IEC 61000-4-21 and the IEEE 299.1 documents. From the results presented in the work, it turns out that the proposed procedure provides more prudent $\mathrm{SE}$ values than the two standards at the low frequencies, especially for standard IEEE 299.1.

It is important to note that the proposed procedure improves the evaluation of the SE of enclosures in IEEE 299.1 at low frequencies by simply using a different calculation. On the other hand, compared to the IEC 61000-4-21 standard, our proposed procedure involves the use of a VNA rather than the PM in the measurement set up. This is certainly an appealing feature, since the VNAs are becoming increasingly widespread and their cost is not higher than that of the PMs (considering that use of the PM involves also the use of a radiofrequency source and of a radiofrequency amplifier).

The proposed measurement approach is supported by results obtained in different nested reverberation chambers located in three different laboratories, namely, at Università Parthenope, Napoli, Italy, at University of Nottingham, Nottingham, U.K., and at Università Politecnica delle Marche, Ancona, Italy.

The paper is organized as follows: in section II, the method is shown; in section III, measurements and results are obtained and discussed; finally, in section IV, the conclusions are drawn along with a further discussion.

\section{RATIONALE OF THE METHOD}

In the IEC 61000-4-21 standard [1, Annex H], the SE is calculated through the following equation:

$$
S E=\frac{\left[P_{\mathrm{r}, \mathrm{o}}\right]^{\mathrm{Max}}}{\left[P_{\mathrm{r}, \mathrm{i}}\right]^{\mathrm{Max}}}
$$

where $P_{\mathrm{r}, \mathrm{o}}$ and $P_{\mathrm{r}, \mathrm{i}}$ are the powers received inside the $\mathrm{RC}$ and enclosure, respectively. The maximums of $P_{\mathrm{r}, \mathrm{o}}$ and $P_{\mathrm{r}, \mathrm{i}}$, which are denoted by the superscript Max in (1), are obtained over one complete cycle of the tuner(s)/stirrer(s). In [1], such powers are directly measured by the PM for all frequencies over the expected measurement FR with no FS and with no correction for impedance mismatch.

In the IEEE 299.1 standard [13, Sec. 7.2], the SE is calculated through the following equation:

$$
S E=\frac{\left\langle P_{\mathrm{r}, \mathrm{o}}\right\rangle_{\text {corr }, \mathrm{N}}}{\left\langle P_{\mathrm{r}, \mathrm{i}}\right\rangle_{\mathrm{corr}, \mathrm{N}}}=\frac{\left\langle\left.\mathrm{S}_{21}\right|_{\text {corr }} ^{2}\right\rangle_{\mathrm{o}, \mathrm{O}, \mathrm{N}}}{\left\langle\left|\mathrm{S}_{21}\right|_{\mathrm{corr}}^{2}\right\rangle_{\mathrm{o}, \mathrm{i}, \mathrm{N}}},
$$

where the symbol $\langle\cdot\rangle_{\mathrm{N}}$ denotes the average value of the powers concerned or equivalently of the squared amplitude of the transmission coefficients $\left(\mathrm{S}_{21}\right)$ over $\mathrm{N}$ uncorrelated samples. The symbol $|\cdot|$ denotes the amplitude value of the parameter concerned. The number of overall samples $\mathrm{N}$ is assumed to be very greater than one. Note that the two power values in (2) can be obtained from the measurements of two coefficients $\mathrm{S}_{21}$, which can be measured by a VNA. Here, it is assumed that a Two-Port VNA is used. When a HS is used, for example MS and FS, the number of samples can be expressed as $\mathrm{N}=\mathrm{n} \cdot \mathrm{k}$, where $\mathrm{n}$ is the number of mechanical positions of the stirrers and $\mathrm{k}$ is the number of frequency points considered for a given FSB. Therefore, when HS is used, \langle\rangle$_{\mathrm{N}}$ denotes the overall 
average value including both MS and FS. The subscript corr indicates that the powers or equivalently transmission coefficients are corrected for impedance mismatch. The subscript $0, i$ indicates that the two antennas concerned are located inside the RC and enclosure, respectively, whereas the subscript 0,0 indicates that the two antennas concerned are positioned both inside the RC. As for the antenna radiation efficiencies, it is specified that they can be considered the typical values according to the standard [1]. Moreover, if the radiation efficiency of the two receiving antennas are equal, the correction is not necessary.

Note that generally an impedance matched antenna is located inside the RC. However, if a receiving antenna having a free-space reflection coefficient less or equal than $-10 \mathrm{~dB}$ is used inside the RC, no correction for impedance mismatch is in practice necessary for such an antenna at the frequencies where the RC is overmoded. If this is the case, only the correction for impedance mismatch for the antenna located inside the enclosure is necessary at low frequencies. Hence, under the assumption that the reflection coefficient of the receiving antenna inside the $\mathrm{RC}$ is less or equal than $-10 \mathrm{~dB}$, (2) can be written as [13]:

$$
\begin{aligned}
S E & =\frac{\left\langle P_{\mathrm{r}, \mathrm{o}}\right\rangle_{\mathrm{corr}, \mathrm{N}}}{\left\langle P_{\mathrm{r}, \mathrm{i}}\right\rangle_{\text {corr }, \mathrm{N}}}=\frac{\left\langle\left.\mathrm{S}_{21}\right|_{\mathrm{unc}} ^{2}\right\rangle_{\mathrm{o}, \mathrm{o}, \mathrm{N}}}{\left\langle\left|\mathrm{S}_{21}\right|_{\mathrm{unc}}^{2}\right\rangle_{\mathrm{o}, \mathrm{i}, \mathrm{N}}} \frac{\left(1-\left|\left\langle\mathrm{S}_{22}\right\rangle_{\mathrm{i}, \mathrm{N}}\right|^{2}\right)}{\left(1-\left.\left|\mathrm{S}_{22}\right\rangle_{\mathrm{o}, \mathrm{N}}\right|^{2}\right)} \\
& \sim \frac{\left\langle\left|\mathrm{S}_{21}\right|_{\mathrm{unc}}^{2}\right\rangle_{\mathrm{o}, \mathrm{o}, \mathrm{N}}}{\left\langle\left|\mathrm{S}_{21}\right|_{\mathrm{unc}}^{2}\right\rangle_{\mathrm{o}, \mathrm{i}, \mathrm{N}}}\left(1-\left|\left\langle\mathrm{S}_{22}\right\rangle_{\mathrm{i}, \mathrm{N}}\right|^{2}\right)
\end{aligned}
$$

where $S_{22, i}$ and $S_{22,0}$ denote the reflection coefficients of the receiving antenna inside the enclosure, which is generally a wall-mounted monopole, and of the one inside the $\mathrm{RC}$, respectively. Note that under the abovementioned assumption, it turns out that $\left\langle\left|\mathrm{S}_{21}\right|_{\mathrm{unc}}^{2}\right\rangle_{\mathrm{o}, \mathrm{o}, \mathrm{N}} \sim\left\langle\left|\mathrm{S}_{21}\right|_{\mathrm{corr}}^{2}\right\rangle_{\mathrm{o}, \mathrm{o}, \mathrm{N}}$. The subscript unc indicates that the relative parameters are uncorrected for impedance mismatch. Note that the non-correction causes an overestimated SE in (3). This occurs also when the maximum values are considered rather than the average ones. Clearly, the correction for impedance mismatch implies the measurements of the reflection coefficient. Generally speaking, the correction should be made for each frequency point. In particular, two cases are of interest: measurement of average and maximum powers, respectively.

When average values of the powers are used for SE and the average mismatch of the antenna does not change considerably over the FSB, the average mismatch values can be used, see (3). This correction method could be applied for any stirring method or their combination.

When the maximum values of the corrected powers are used for SE calculation, the average mismatch values could still be used for the correction regardless of the stirring method, if a possible reduction of the quality of the approximation is accepted. This is confirmed by measurement results presented in the next Section. The validity of this approximate method of correction for impedance mismatch is achieved by comparing results from uncorrected transmissions coefficient with the ones from corrected transmission coefficients.
Before expressing (1) in terms of samples of powers or equivalently of $\left|S_{21}\right|_{0,0}^{2}$ and $\left|S_{21}\right|_{0, i}^{2}$, the statistical distributions of $P_{\mathrm{r}, \mathrm{i}}$ and $\left|\mathrm{S}_{21}\right|_{\mathrm{o}, \mathrm{i}}^{2}$ are discussed. In [20], the distribution of the power $P_{\mathrm{r}, \mathrm{i}}$ when the system is fed by outer chamber was shown. For an enclosure under test in the RC, at least a few small apertures can certainly be considered. Hence, they are sufficient to make $P_{\mathrm{r}, \mathrm{i}}$, or equivalently $\left|\mathrm{S}_{21}\right|_{\mathrm{o}, \mathrm{i}}^{2}$, exponentially distributed. Such a distribution is supported by results from the Chi-Square Goodness-of-Fit test applied to measured samples of $\left|\mathrm{S}_{21}\right|_{\text {corr,o,i }}^{2}$. It is found that the hypothesis $\mathrm{H}_{0}$ is not rejected to the significance of 0.05 from minimum working frequency $\left(\mathrm{f}_{60}\right)$ as detailed on the measurements used for tests in the next section. However, it is specified that the extreme values elude any Goodness-of-Fit test. In fact, it is experimentally found that $\left|S_{21}\right|_{\text {corr,o,o }}^{2}$ and $\left|S_{21}\right|_{\text {corr,oi }}^{2}$ have the same distribution type, which is a chi-squared distribution with two degrees of freedom (exponential distribution), except for some extreme values that are present in samples of $\left|\mathrm{S}_{21}\right|_{\mathrm{o}, \mathrm{i}}^{2}$ at low working frequencies where the number of modes is low. As shown in the next section, at low frequencies, such extreme values play a key role in the accurate calculation of the SE of enclosures by NRCs.

In the IEC 61000-4-21 standard [1], the maximums of $P_{\mathrm{r}, \mathrm{o}}$ and $P_{\mathrm{r}, \mathrm{i}}$ in (1) are obtained over one complete cycle of the tuner(s)/stirrer(s) as described above. However, another approach is possible. Indeed, one can think to use a sufficiently large number $(\mathrm{N})$ of uncorrelated samples, regardless of the adopted stirring technique. In particular, when only MS from metallic stirrer(s) is used and the samples are measured for all the independent positions of the stirrer(s), the two approaches are equivalent. The rationale is valid independently of the stirring method for large $\mathrm{N}$ values; i.e., the use of samples from HS, such as a combination of MS and FS, improves results even when compared to those achieved from the complete cycle of the tuner(s)/stirrer(s). In fact, the larger $\mathrm{N}$ the more results improve. This is supported by the extreme value theory (EVT) [21], which has also been applied to the maximum field in an RC [22]-[25]; further, in [26] an upper bound is rightly considered for field and power, as well as in [27] where a deterministic approach is used. More recent work has focused on the use of EVT to model the RC field behavior near the walls [28], where HS was used to enlarge the statistical ensemble, the field to wire coupling [29], and the accurate characterization of the nested chamber field [30]. Interesting applications include high-power susceptibility testing [31]. Actually, the coefficient of variation of the distribution of the exceedance over certain thresholds for both $P_{\mathrm{r}, \mathrm{o}}$ and $P_{\mathrm{r}, \mathrm{i}}$, is well approximated by the generalized Pareto distribution for large thresholds [21], [32]-[33]. The exceedance decreases with the increasing threshold. This implies that possible differences between the values of the ratios calculated using the average and maximum values can be appreciated only for large $\mathrm{N}$ values, where statistical fluctuations do not mask them. In particular, it is experimentally found that these differences are not negligible when the number of processed uncorrelated 
samples is greater than 200. Specifically, we increased gradually the number of processed uncorrelated samples until the differences (occurring at low frequencies) between the values of the ratios calculated using the average and maximum values become significant and essentially stable. Note that real FSB are compatible with necessary number of uncorrelated samples; in any case, one notes that the FSB cannot be taken too wide in order to ensure that no bias is applied to SE.

Since the correction for impedance mismatch results in a possible underestimation of the SE, we can intentionally and safely consider the powers in [1] as powers corrected for impedance mismatch without loss of generality. Therefore, by considering all of the above, and by assuming again that a receiving antenna having a free-space reflection coefficient less or equal than $-10 \mathrm{~dB}$ is used inside the $\mathrm{RC}$, (1) can be written as follows:

$$
\begin{aligned}
S E & =\frac{\left[P_{\mathrm{r}, \mathrm{o}}\right]^{\mathrm{Max}}}{\left[P_{\mathrm{r}, \mathrm{i}}\right]^{\mathrm{Max}}} \equiv \frac{\left[P_{\mathrm{r}, \mathrm{o}}\right]_{\mathrm{corr}, \mathrm{N}}^{\mathrm{Max}}}{\left[P_{\mathrm{r}, \mathrm{in}}\right]_{\mathrm{corr}, \mathrm{N}}^{\mathrm{Max}}} \\
= & \frac{\left[\left|\mathrm{S}_{21}\right|_{\mathrm{unc}}^{2}\right]_{\mathrm{o}, \mathrm{o}, \mathrm{N}}^{\mathrm{Max}}}{\left[\left|\mathrm{S}_{21}\right|_{\mathrm{unc}}^{2}\right]_{\mathrm{o}, \mathrm{i}, \mathrm{N}}^{\mathrm{Max}}} \frac{\left(1-\left|\left\langle\mathrm{S}_{22}\right\rangle_{\mathrm{i}, \mathrm{N}}\right|^{2}\right)}{\left(1-\left|\left\langle\mathrm{S}_{22}\right\rangle_{\mathrm{o}, \mathrm{N}}\right|^{2}\right)} \\
& \sim \frac{\left[\left|\mathrm{S}_{21}\right|_{\mathrm{unc}}^{2}\right]_{\mathrm{o}, \mathrm{o}, \mathrm{N}}^{\mathrm{Max}}}{\left[\left|\mathrm{S}_{21}\right|_{\mathrm{unc}}^{2}\right]_{\mathrm{o}, \mathrm{i}, \mathrm{N}}^{\mathrm{Max}}}
\end{aligned}
$$

Where $N>1$. In (4), it is highlighted that the maximum values are achieved from $\mathrm{N}$ uncorrelated samples and the average mismatch is considered for correction. It is noted that the non-correction causes an overestimated SE in (4) as well.

Finally, it is important to note that the use of maximum values makes the SE evaluation fluctuate more than the use of average values. In fact, the distribution of extreme values has a greater dispersion than the distribution of average values. Summing up, to measure the SE of enclosures in $\mathrm{RC}$ we propose to:

- use eq. (4),

- exploit the HS including both MS and FS to obtain the power measurements involved in (4),

- possibly use a wall-mounted monopole inside the enclosure,

thus merging in a single procedure the two standard procedures defined in the IEC 61000-4-21 and IEEE 299.1. As shown in the following sections, this proposed procedure provides more prudent SE values than the two standards at the low frequencies.

\section{A. Short discussion on $M U$}

The MU, which is here understood as a combined and expanded MU [34], can be calculated by using the MUs of the parameters involved in the measurement model and the law of propagation of the uncertainties by considering type A and B uncertainties [34]-[35]. MUs of the involved parameters can be empirically achieved by measurements [8], as well as their distributions. The mean values of such parameters have approximately normal distribution. For exponential distributions of the parameters $\left|S_{21}\right|_{\text {corr;o,o }}^{2}$ and $\left|S_{21}\right|_{\text {corr;o,i }}^{2}$, the distribution of the normalized maxima can easily be achieved from EVT [23], [25]. In [36], the distribution of the ratio between two normalized exponential distributions was described.

However, any procedure to calculate the MUs of SE given in (3) and (4) has to include the non-uniformity of the field in the enclosure at the low frequencies where the modal density is low. This is normally very difficult to be achieved by measurements because of sizes of enclosures. In fact, it involves moving the receiving antennas into the volume of the enclosure. Work is still necessary to address such an uncertainty component [13, Sect. 6]. The focus of the paper is not the calculation MU. In any case, the condition on $\mathrm{f}_{60}$ and the use of FS, reduce the spatial non-uniformity of the field; therefore, the method gives a very high confidence that an enclosure provides the measured SE [13, Sec. 7.2.1].

Finally, it is important to highlight that the dispersion of the maximums normalized to the average value inside the enclosure is greater of that of the ones inside the $\mathrm{RC}$ at low frequencies according to the $\mathrm{SE}$ value. This is because the $\mathrm{RC}$ works in overmoded conditions over the working FR whereas the enclosure is overmoded only to high frequencies.

\section{MEASUREMENTS RESULTS}

In this section, measurements and results from experiments realized at different laboratories are shown. Hence, different chambers and stirring techniques are used. Specifically, results from measurements performed at Università Parthenope, Napoli, Italy, at University of Nottingham, Nottingham, England, and at Università Politecnica delle Marche, Ancona, Italy are shown. It is specified that all uncorrelated samples from such measurements can be considered independent as well.

\section{A. Measurement results obtained at University "Parthenope", Napoli, Italy}

The measurements were conducted in RC at the Università Parthenope. Measurements were performed for the work of [37] and for another paper [16], where however the aims were different from that of this paper. The $\mathrm{RC}$ used for the measurements is a cubic chamber of $8 \mathrm{~m}^{3}$ volume, where the input electromagnetic field is randomized by means of three metallic stirrers rotating in continuous mode. Random MS due to the vibrations of the chamber walls under the effect of the motors of the stirrers adds to the regular MS so that a very large number of uncorrelated samples can be acquired. It must be noted that the non-correlation of the acquired samples was verified by the autocorrelation function and a threshold of $1 / e$. It is specified that this RC is overmoded at about $1 \mathrm{GHz}$, i.e., to such a frequency $\left|S_{21}\right|_{0,0}^{2}$ fits a chi squared $\left(\chi^{2}\right)$ distribution.

Two different series of measurements are available, which concern two different enclosures. One series concerns an enclosure that has dimensions of $0.30 \mathrm{~m} \times 0.30 \mathrm{~m} \times 0.30 \mathrm{~m}$ and the other has dimensions of $0.49 \mathrm{~m} \times 0.49 \mathrm{~m} \times 0.49 \mathrm{~m}$. They are also made of different materials as detailed below. For both cases, a $24 \mathrm{~mm}$ monopole antenna is placed on one interior 
wall of the enclosure [16], [37]. The same measurement set up includes two double-ridge waveguide horn antennas and a two-port vector network analyzer (VNA), model Agilent 8363B PNA. The intermediate frequency bandwidth (IFBW) and source power are set to $3 \mathrm{kHz}$ and $0 \mathrm{dBm}$, respectively.

We consider the smaller enclosure first. It wss placed on foam support within the RC during the measurements; the clearance from the chamber floor was about $50 \mathrm{~cm}$. Note that $\mathrm{f}_{60}$ for this enclosure is $1.927 \mathrm{GHz}$. The enclosure has an interchangeable wall. For the measurements used in this paper, the interchangeable wall was an aluminium wall accurately blocked by an adhesive aluminium tape. For this enclosure only MS is used, both inside the RC and the enclosure. Inside the enclosure a single stirrer rotating in continuous mode is used. Fig. 1 shows the inside of the RC where the enclosure is visible as well.

The FR is from $2 \mathrm{GHz}$ to $10 \mathrm{GHz}$, with step of $1 \mathrm{GHz}$. Consequently, each frequency sweep includes 9 frequency points. During the continuous stirring, where all stirrers were operated, 1000 uncorrelated samples are automatically acquired at each frequency point in as many sweep frequency and for each of the measured coefficient.

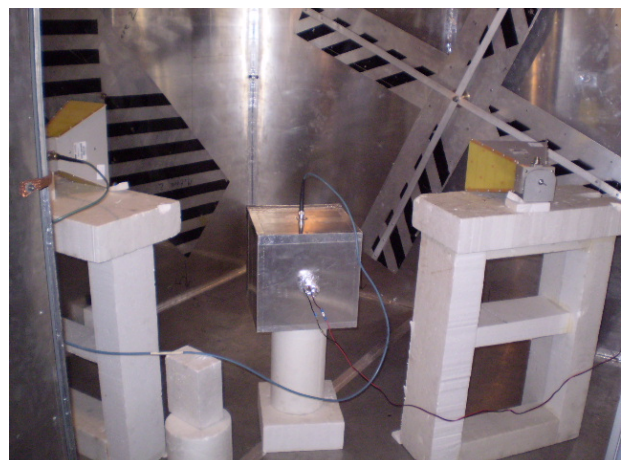

Fig. 1. Inside of the RC at University of Naples. The enclosure is visible in the chamber, as well as the motor that drives the stirrer inside the enclosure.

The transmission coefficients $\left[S_{21}\right]_{\mathrm{o}, \mathrm{o}}$, and $\left[S_{21}\right]_{\mathrm{o}, \mathrm{o}}$, as well as the reflection coefficient $\left[S_{22}\right]_{\mathrm{i}}$ were measured.

In order to show the damping effect of the resonance inside the enclosure, measurements were performed both with the unloaded and loaded enclosure. The load was a small piece (4 $\mathrm{cm} \times 4 \mathrm{~cm} \times 8 \mathrm{~cm}$ ) of microwave absorption material used for walls in anechoic chambers. It is positioned on foam support at center of a side within the enclosure during the relative measurements; the clearance from the side is about $7 \mathrm{~cm}$.

It is verified by Chi-Square Goodness-of-Fit test that the measured samples of $\left|S_{21}\right|_{0, i}^{2}$ have an exponential distribution to the significance of 0.05 at the smallest acquired frequency $\mathrm{f}_{\min }=2 \mathrm{GHz}$.

Fig. 2 shows the SE of the enclosure, both when the correction for impedance mismatch of the monopole antenna is performed and when it is not performed.

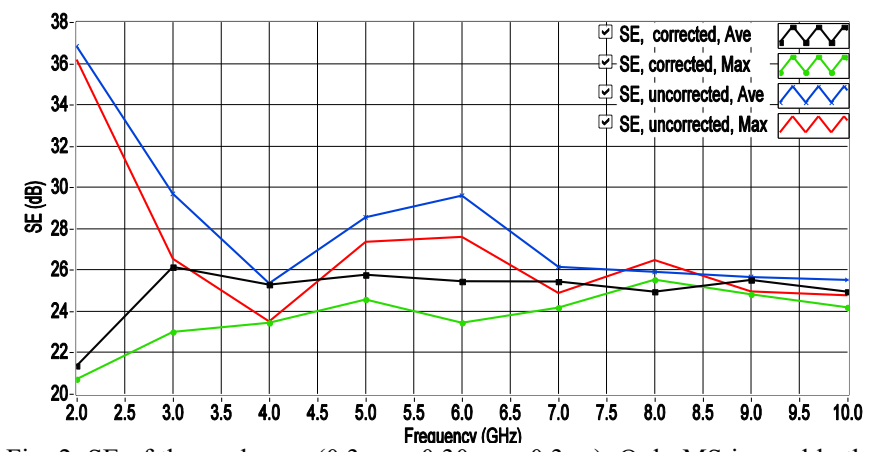

Fig. 2. SE of the enclosure $(0.3 \mathrm{~m} \times 0.30 \mathrm{~m} \times 0.3 \mathrm{~m})$. Only MS is used both inside the RC and the enclosure. Black and square-marked trace and blue and cross-marked trace are related to average values and corrected and uncorrected for impedance mismatch of the monopole antenna, respectively. Green and circle-marked trace and red and unmarked trace are related to max values and to corrected and uncorrected for impedance mismatch of the monopole antenna, respectively.

Note that for frequencies up to $7 \mathrm{GHz}$ the green trace, obtained by using maximum values of the measured powers, is clearly lower than the black trace, obtained by using average values of the measured powers. It is noted that in both cases the correction for impedance mismatch of the monopole antenna was performed. Similarly, for frequencies up to 7 $\mathrm{GHz}$, the red trace, obtained by using maximum values of the measured powers, is clearly lower than the blue one, obtained by using average values of the measured powers. It is noted that in the latter two cases the correction for impedance mismatch of the monopole antenna was not performed.

From all measurement results achieved for this paper, which are not all shown for brevity, it has been seen that the number of uncorrelated samples $\mathrm{N}$ has to be greater than 200 in order that the differences under consideration are essentially stable. They also include the position (that includes the polarization) of the receiving antenna that could not pick up the field for specific resonances. However, this can affect results at very low frequencies; but, such an impact is acceptable to frequencies greater or equal then $\mathrm{f}_{60}$. In any case, the MU due to the correction for impedance mismatch depends on the mismatch level [38]. Note that when the mismatch is strong (close to one) a small error on $\left[S_{22}\right]_{i}$ produces a considerable error on the SE. It could justify the smaller difference between the traces at the frequency of $2 \mathrm{GHz}$, where a greater difference is expected.

Summing up, Fig. 2 shows that the use of the ratios between the maximums (rather than the averages) of the measured powers provides more prudent values of SE of enclosures at the low frequencies. It is likely that this occurs particularly when low internal losses are present inside the enclosure. Indeed, the losses damp each internal resonance according to their amount and relative coupling. To better show this effect, we have added losses inside the enclosure. It is specified that the effect of the resonance is however damped as the antennas inside the enclosure load the enclosure itself according to their reflection coefficient. Therefore, a wall-mounted monopole antenna negligibly loads the enclosure when it is considerably mismatched. On the other hand, in real-life scenarios, the equipment of the enclosure represents a certain load for the enclosure itself. In Fig. 3, one can note that the differences observed in Fig. 2, are clearly reduced by the addition of 
losses. It further proves that such differences are due to the resonance phenomenon.

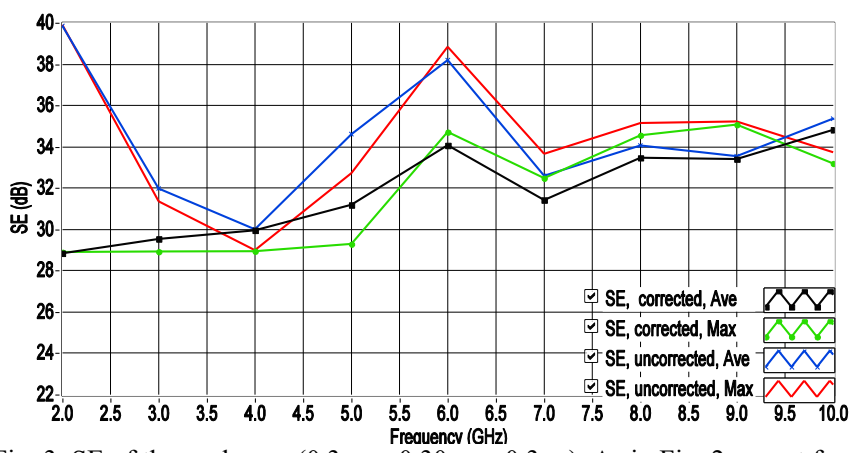

Fig. 3. SE of the enclosure $(0.3 \mathrm{~m} \times 0.30 \mathrm{~m} \times 0.3 \mathrm{~m})$. As in Fig. 2 except for the presence of a small absorber inside the enclosure.

Results from measurements performed for the other enclosure are also shown as they are very meaningful. In fact, these measurements were performed for an enclosure of metallic grid whose losses depend naturally on the frequency. The enclosure is a cubic box of $49 \mathrm{~cm}$ side size. Specifically, the enclosure is made with metallic grid and its mesh size is 1 $\mathrm{mm}$ : it has a foam structure as a support, as Fig. 4 shows. It was placed on foam support within the RC during the measurements. The clearance from the chamber floor is $50 \mathrm{~cm}$. Again a $24 \mathrm{~mm}$ monopole antenna is placed on one interior wall of the enclosure. It is verified by Chi-Square Goodnessof-Fit test that the measured samples of $\left|S_{21}\right|_{0, i}^{2}$ have an exponential distribution to the significance of 0.05 from the lowest acquired frequency $\mathrm{f}_{\min }=1 \mathrm{GHz}$. Note that $\mathrm{f}_{60}$ for this enclosure is $0.93 \mathrm{GHz}$. For this case, only MS is used inside the $\mathrm{RC}$; the enclosure is randomly and uniformly fed from its whole surface. The same RC and measurement set-up were used for these measurements. It is shown in [16], and it is also shown in Fig. 4.

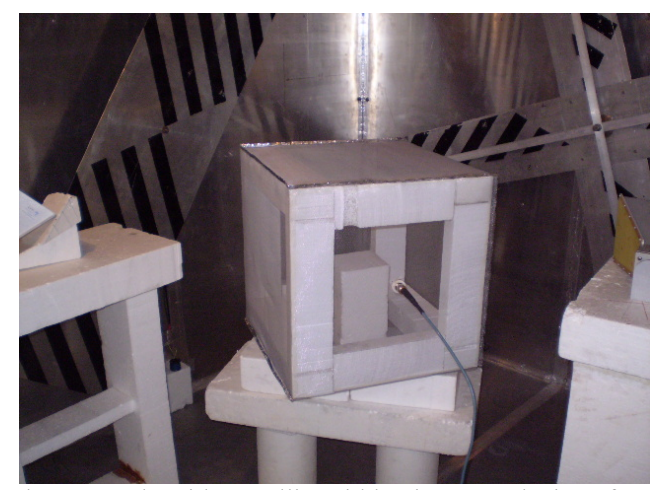

Fig. 4. Enclosure made with metallic grid having a mesh size of $1 \mathrm{~mm}$ inside the chamber.

The FR is from $1 \mathrm{GHz}$ to $18 \mathrm{GHz}$, with step of $0.5 \mathrm{GHz}$. Consequently, each frequency sweep includes 35 frequency points. 3000 uncorrelated samples were automatically acquired at each frequency point in as many sweep frequency and for each of the measured coefficient.

$\mathrm{SE}$ of this enclosure is shown in [16, Fig. 6], when it is calculated by the ratio of average values. In Fig. 5, the SE of the enclosure obtained from average and maximum values is shown. Results are shown up to frequency $6 \mathrm{GHz}$ even though no useful information is included to the frequencies greater than $3 \mathrm{GHz}$ for the aims of this paper. It is not shown the case where the SE was not corrected for impedance mismatch of the monopole inside the enclosure as it simply still confirms the results as mentioned above.

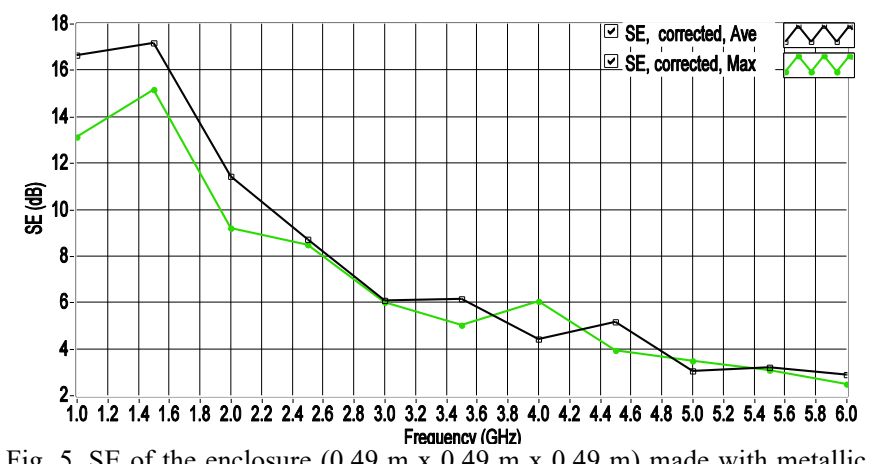

Fig. 5. SE of the enclosure $(0.49 \mathrm{~m} \times 0.49 \mathrm{~m} \times 0.49 \mathrm{~m})$ made with metallic grid of mesh size is $1 \mathrm{~mm}$. Only MS is used inside the RC; the enclosure is randomly and uniformly fed from its whole surface. Black and square-marked trace is related to average values. Green and circle-marked trace is related to maximum values. The two traces are corrected for impedance mismatch of the monopole antenna inside the enclosure.

It is important to note that the differences between the two evaluated SE are still clearly visible at low frequencies even though they damp down due to the losses that increase rapidly with the increasing of the frequency. In fact, even though the mesh size is very much smaller than wavelength, its wires however scatter energy through the wall of the enclosure. This results in a rapid increase of the losses inside the enclosure with the increasing of the frequency. Consequently, the resonances are rapidly damped as the frequency increases.

It is also important to note that the use of HS tends to increase the differences in SE results shown in the Figs 2-3 and Fig. 5 at low frequencies, as explained above and as we can see from results shown in the next Section.

\section{B. Measurement results obtained at University of Nottingham, Nottingham, England.}

Measurements acquired in the RC at University of Nottingham for results in [39] are reused here for our current purpose. The RC has dimensions of $(4.84 \times 3.72 \times 3.11) \mathrm{m}^{3}$ and the enclosure has dimensions of $(0.36 \times 0.45 \times 0.54) \mathrm{m}^{3}$. Note that the inner chamber in [39] is the enclosure in this paper. It has an aperture, whose size is $0.05 \mathrm{~m} \times 0.05 \mathrm{~m}$, which is closed both with a 'copper mesh' and with a metallic plate for tests. Hence, three configurations of the enclosure are considered: one where the aperture is closed by a metallic plate, one where the aperture is closed by a 'copper mesh', and in the third case, the aperture is open. They are denoted by conf. 1 , conf. 2 , and conf. 3 , respectively. The $\mathrm{f}_{60}$ for this enclosure is $1.3 \mathrm{GHz}$.

Inside the outer chamber, the input electromagnetic field is randomized by means of HS using one big metallic stirrer, which works in step mode for measurements used in this paper, and FS; inside the enclosure, only FS is used. The measurement setup includes a two-port VNA, model Agilent PNA E8362B and three antennas. The two receiving antennas, one of which is inside the $\mathrm{RC}$ and the other one inside the enclosure, are two equal double ridge horn antennas, model 
AH systems SAS-571. The third antenna is an ETS Lindgren double-ridged horn antenna, model 3115 , which is used as a transmitting antenna in the outer chamber. The impedance mismatch and the radiation efficiency of the two antennas inside the $\mathrm{RC}$ can be considered equal. The inside of the $\mathrm{RC}$ is shown in Fig. 6. Measurements were performed for 120 positions of the stirrer (mechanical positions), which were evenly spaced in angle. For each stirrer position 16,001 point in frequency were acquired by the VNA. The FR was set from 1 to $20 \mathrm{GHz}$; the intermediate frequency bandwidth was set to $3 \mathrm{kHz}$; the port power was set to $0 \mathrm{dBm}$. The step frequency (SF) was $1.1875 \mathrm{MHz}$. The transmission coefficient inside the outer chamber $\left(\left[S_{21}\right]_{0,0}\right)$ and that between outer and inner chambers $\left(\left[S_{21}\right]_{0, i}\right)$ were measured for the three abovementioned configurations. In this case, measurements of the reflection coefficient $\left(\left[S_{22}\right]_{i}\right)$, which corresponds to the horn antenna within the enclosure, are not available as they were not acquired during measurements for the purposes of [39]. Therefore, measurements are not corrected for the possible appreciable impedance mismatch at the low frequencies of the horn antennas placed within the enclosure. It does not absolutely affect the results focused in this paper and, however, such a correction could be remarkable at very low frequency as the antenna is impedance matched in free space.

No further measurements are available at the moment for this RC; however, according to the measurements which are available, it is established that it is certainly overmoded at a frequency of $1 \mathrm{GHz}$.

It is verified by Chi-Square Goodness-of-Fit test that the measured samples of $\left|S_{21}\right|_{0, i}^{2}$ have an exponential distribution from the smallest acquired frequency $\mathrm{f}_{\min }=1 \mathrm{GHz}\left(\mathrm{f}_{60}=1.3\right.$ $\mathrm{GHz}$ ) to the significance of 0.05 .

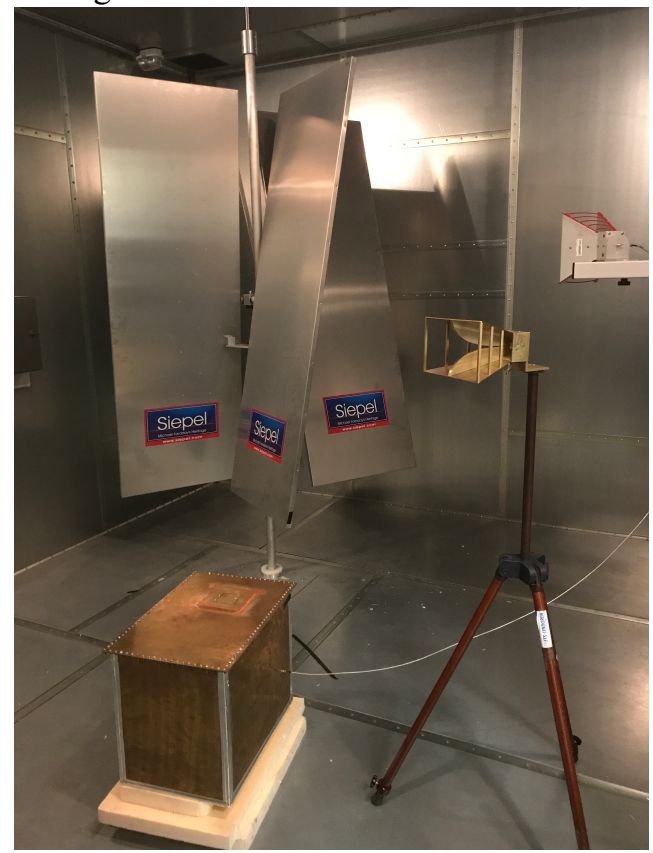

Fig. 6. Inside of the RC at University of Nottingham. The relative enclosure is well-visible inside the chamber.

The measurements were performed by using the three abovementioned configurations. It is specified that the non- correlation of the samples related to FS was verified by the autocorrelation coefficient considering the samples over the FSB. The SE is calculated by using two ways of stirring: in the first only FS is present, i.e., we have a single position of the stirrers (single frequency sweep) and FS includes 200 samples that correspond to a FSB of about $235.8 \mathrm{MHz}$. In the second, the MS includes 120 positions of the stirrers (120 frequency sweep) and FS includes 200 samples again. It is specified that here the frequency averaging process is performed by sliding the FSB window to step equal to the FSB itself and the obtained maximum or average value is represented at relative center. Clearly, when HS is used, the relative maximum or average value is based on the total number of samples. It is important to note that the FSB cannot be taken too wide to ensure no bias is applied to SE due to $\left|\mathrm{S}_{21}\right|_{\mathrm{o}, \mathrm{o}}^{2}$ and $\left|\mathrm{S}_{21}\right|_{\mathrm{o}, \mathrm{i}}^{2}$ dependence on frequency.

Fig 7, Fig. 8, and Fig. 9 show SE of the enclosure for the three abovementioned configurations, respectively. It is calculated for both of the two abovementioned combinations of stirring and by both the ratio of the maximum values and average values. Specifically, it is confirmed that SE obtained from the ratio between the maximum values is lower at the low frequencies for the effect of the resonances inside the enclosure. This is observed in spite of the greater randomness of the maximum values. From such Figs, again we see that this phenomenon is all the more marked and extends in frequency the lower the losses inside the enclosure including the leakage.

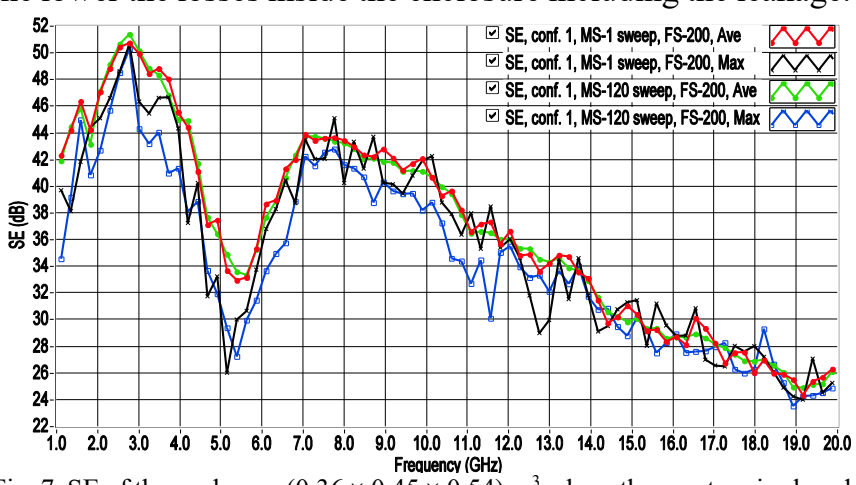

Fig. 7. SE of the enclosure $(0.36 \times 0.45 \times 0.54) \mathrm{m}^{3}$ where the aperture is closed by e metallic slab (conf. 1). All traces are uncorrected for impedance mismatch of the horn antenna inside the enclosure. Red and circle-marked trace and black and cross-marked trace are related to average and maximum values, respectively, as well as to FS only, with a FSB of $235.8 \mathrm{MHz}$ (200 samples). Green and circle-marked trace and blue and square-marked trace are related to average and maximum values, respectively, as well as to HS including 120 samples from MS and 200 samples from FS that correspond to the FSB of $235.8 \mathrm{MHz}$ again.

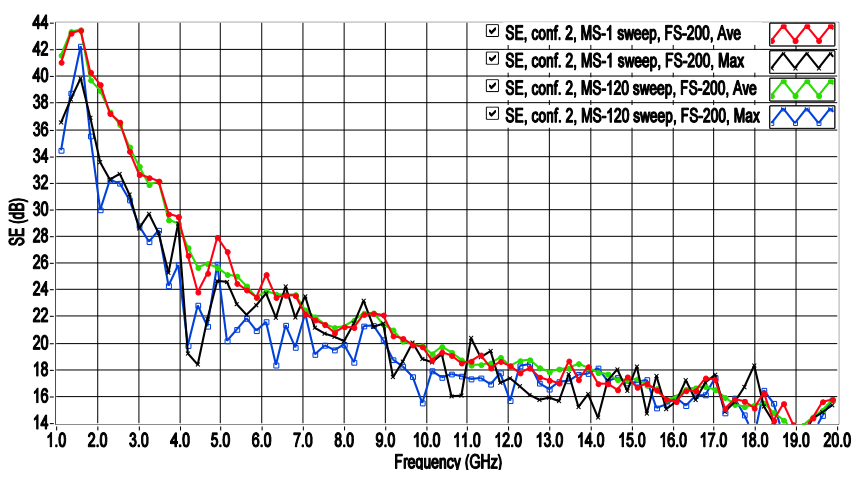


Fig. 8. SE of the enclosure $(0.36 \times 0.45 \times 0.54) \mathrm{m}^{3}$. As Fig. 7 except for the fact that these results are related to the conf. 2 , where the aperture is closed by copper mesh.

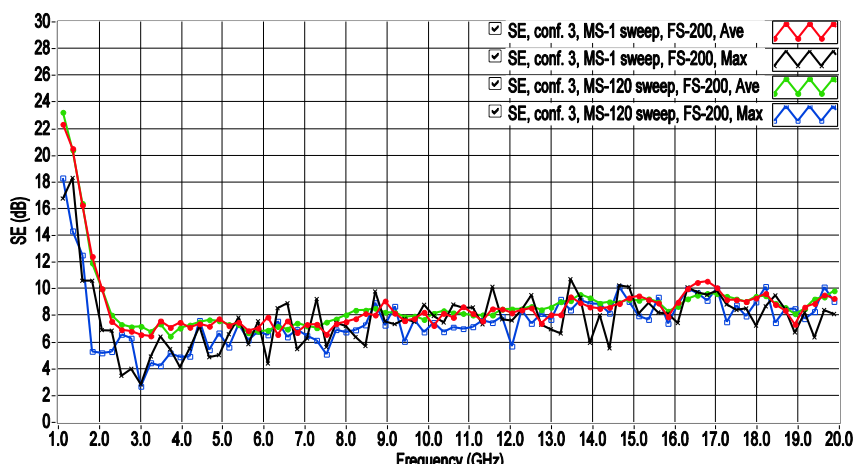

Fig. 9. SE of the enclosure $(0.36 \times 0.45 \times 0.54) \mathrm{m}^{3}$. As Fig. 7 except for the fact that these results are related to the conf. 3 , where the aperture, whose size is $0.05 \mathrm{~m} \times 0.05 \mathrm{~m}$, is open. The cut-off effect of the aperture is clearly visible here.

Note that the cut-off effect of the aperture is clearly visible in Fig. 9. We note that measurement results from HS including FS confirm the theory again as well as the ones observed from previous results where only MS were present.

\section{C.Measurement results obtained at University of Ancona, Italy.}

The RC of the Università Politecnica delle Marche has dimensions $6 \mathrm{~m} \times 4 \mathrm{~m} \times 2.5 \mathrm{~m}$. It is equipped by a vertical zfolded stirrer and a horizontal helical stirrer [40]. Inside this outer chamber there is an enclosure having dimensions $1.2 \mathrm{~m}$ x $0.8 \mathrm{~m} \mathrm{x} 0.9 \mathrm{~m}$. The $\mathrm{f}_{60}$ for this enclosure is $0.7 \mathrm{GHz}$. The upper wall hosts a rectangular aperture typically adopted for SE measurements of materials. For aims of this paper, inside the RC, the input electromagnetic field is randomized by means of HS using only horizontal stirrer, which works in step mode for measurements used in this paper, and FS; only 9 equispaced positions for the MS are considered. It is specified that this RC is overmoded at about $500 \mathrm{MHz}$. Inside the enclosure, only FS is used. Measurements were carried out by a four port VNA (Agilent E5071B) connected to two logperiodic antennas in the RC (Schwarzbeck Mess-Elektronik USLP 9143) and two antennas inside the enclosure. The latter two antennas are a double ridged horn antenna from $\mathrm{AH}$ Systems, model SAS-571 and a homemade discone antenna, which works well in the FR from $1 \mathrm{GHz}$ to $10 \mathrm{GHz}$. It is specified that inside the enclosure are present two antennas because the same measurement set-up for SE of material is considered [39]. Actually, only measurements from the horn antenna are used; that is, the horn antenna has to be considered as the receiving antenna inside the enclosure. However, the loading of two antennas present inside the enclosure, whose effect is not negligible at low frequencies has to be considered.

The investigated FR was $800 \mathrm{MHz}-8.4 \mathrm{GHz}$, with a SF of $250 \mathrm{kHz}$. Hence, for each MS position, the whole FR was investigated according to above defined SF. The intermediate frequency bandwidth was set to $100 \mathrm{~Hz}$. Figure 10 shows a photo of the RC internal view.

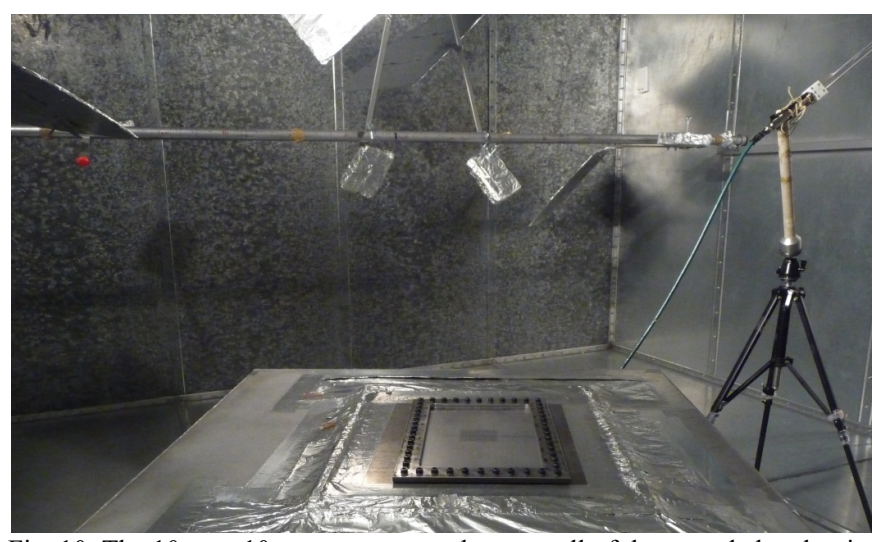

Fig. 10. The $10 \mathrm{~cm} \times 10 \mathrm{~cm}$ aperture on the top wall of the nested chamber in the Ancona's RC.

It is specified that the FSB used for data processing is 400 frequency points, which corresponds to $100 \mathrm{MHz}$. Again, the non-correlation of the samples related to FS was verified by the autocorrelation coefficient considering the samples over the FSB.

The transmission coefficient inside the outer chamber $\left(\left[S_{21}\right]_{0,0}\right)$ and that between outer and inner chambers $\left(\left[S_{21}\right]_{0, \mathrm{i}}\right)$ were measured. Measurements were not corrected for the reflection coefficient of the adopted antennas, because data was not acquired. However, the antennas are well matched in free space in the considered FR. Therefore, the correction can be neglected for antennas inside the $\mathrm{RC}$ whereas for the receiving antenna inside the enclosure, the correction, which can be necessary to the low frequencies, does not affect conclusions as specified above. The radiation efficiencies considered for the calculation of SE are 0.75 for the two Log Periodic antennas and 0.9 for the horn antenna [1]. The radiation efficiencies are roughly considered constant in the measurement FR [1]. Again, it is verified that the samples of $\left|\mathrm{S}_{21}\right|_{\mathrm{o}, \mathrm{i}}^{2}$ have an exponential distribution to the significance of 0.05 from the smaller acquired frequency $f_{\min }=800 \mathrm{MHz}$. Three configurations of the enclosure were considered: one where the aperture having dimension $10 \mathrm{~cm} \times 10 \mathrm{~cm}$ is completely closed by an aluminum sheet, one where the same aperture is covered by an aluminum mesh, and the third where the aperture is completely open [39]. They are denoted by conf. 1 , conf. 2 , and conf. 3 , respectively. The wire mesh is diamond-shaped and has all four sides equal, of length is 2.4 $\mathrm{mm}$; the thickness of the aluminum wire is $0.5 \mathrm{~mm}$. Measurements were also repeated by inserting a small piece of absorbing material (one pyramidal absorber Eccosorb VHP-8NRL by Emerson \& Cuming) inside the enclosure, in order to compare the results to the case with no added load as mentioned above. 


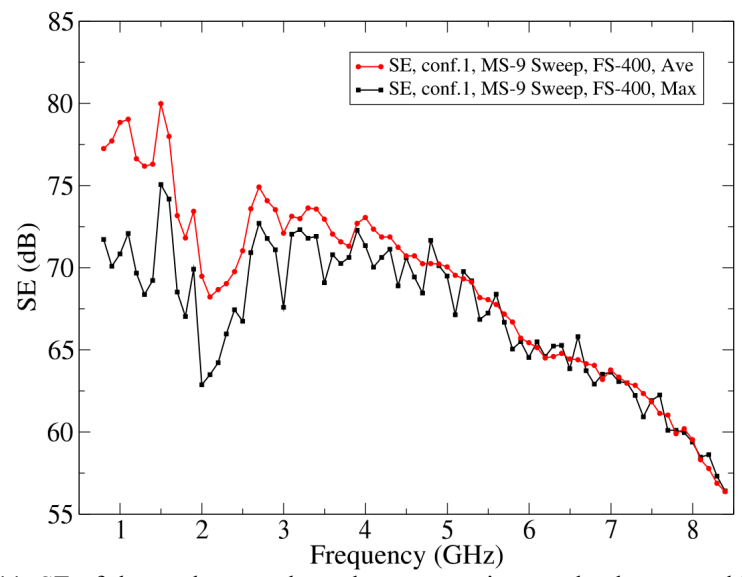

Fig. 11. SE of the enclosure where the aperture is completely covered by an aluminum sheet.

Figures 11-14 report the measured SE of the enclosure by eqs. (3) and (4). Each trace is achieved by combining both the FS (400 frequency points inside the FSB) and the MS (repeating the whole measurements for 9 stirrer angles). We note that the averaged values denote a larger value for the SE up to about $4 \mathrm{GHz}$ whereas above this frequency value the curves derived from different methods almost overlap.

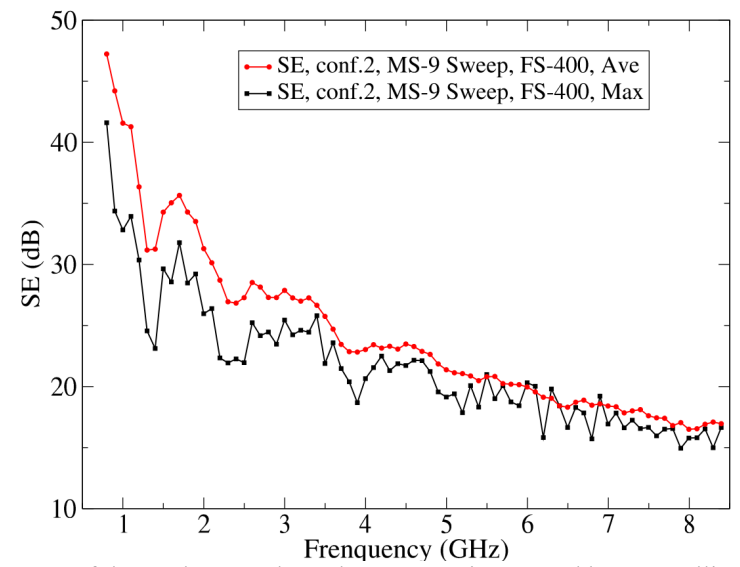

Fig. 12. SE of the enclosure where the aperture is covered by a metallic mesh.

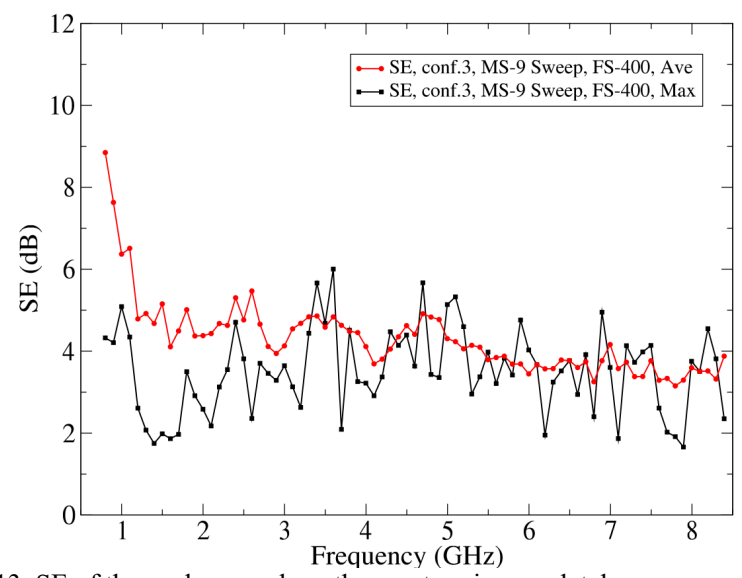

Fig. 13. SE of the enclosure where the aperture is completely open.

Note that the configurations of the enclosure considered in Figs. 11-13 are different; therefore, the obtained SE are different. Apart from the greater statistical fluctuations due to the maximum values, the difference of about $5 \mathrm{~dB}$ at the lowest frequency point in Fig. 13 where the aperture is open could be favoured by the shielding effect for cut-off of the aperture itself along with the position of the receiving antenna inside the enclosure that closely couples with the resonance. It is specified that such a difference is the greatest one achieved for the nine positions of MS in the RC. Indeed, by referring to the power ratios considered in the work, at the lower frequencies (where the modal density is low) the maximums relevant to the $\mathrm{RC}$ (which is always overmoded over the working FR) are less dispersed than the maximums relevant to the enclosure. Therefore, at the low frequencies, the maximums relevant to the enclosures dominate the abovementioned ratios. It is also noted that the HS (including FS) excites more resonance (at the low frequencies) than MS on its own, and this improves the evaluation of the SE as mentioned above. The more the frequency decreases and the number of uncorrelated samples increases the more the differences tend to increase.

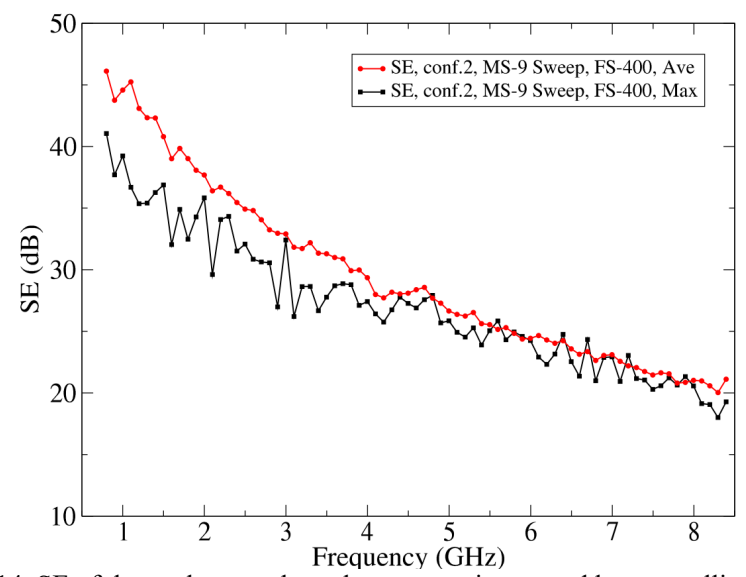

Fig. 14. SE of the enclosure where the aperture is covered by a metallic mesh and a pyramidal absorber is placed inside.

From Fig. 14, we note that the addition of the specified load smooths the traces and reduces their differences in SE at the low frequencies but it does not strongly reduce the differences because it likely is not greater than the load due to the two antennas inside the enclosure and to its wall. Note that the differences tend to increase with an increase in the number of processed uncorrelated samples. Moreover, we note that considering the averaged values gives rise to smaller fluctuations for the SE. Ultimately, these results confirm again that at the low frequencies the resonances inside the enclosure call for a SE calculated by the ratio between maximum values of the powers.

\section{DISCUSSION AND CONCLUSIONS}

In this work, the two procedures for the SE measurement of enclosures in RC defined in the IEEE 299.1 and the IEC 61000-4-21 standards are merged in a single enhanced one, which exploits hybrid stirring, uses the maximum values of measured powers and possibly exploits a wall-mounted monopole inside the enclosure.

The proposed approach and the already available ones have been compared and classified in terms of quantities adopted for the computation (average and maximum received power) and of the exploited stirring technique (MS and FS). 
In the lower FR, the $\mathrm{SE}$ computation through the maximum received power returns a lower value with respect to that achieved exploiting average powers. Differences are typically well appreciable up to some multiple (3-5) of $\mathrm{f}_{60}$ according to the losses inside the enclosure and when the number of processed uncorrelated samples is large. In particular, we have experimentally found that this number must be greater than 200, regardless of the adopted stirring technique. The lower the losses inside the enclosure, the more extended in frequency the phenomenon is. This is due to the resonances in the enclosure under test (low modal density). This result is confirmed by adopting RCs having different dimensions and used in different laboratories. Moreover, it is confirmed for different enclosure dimensions, also including different aperture dimensions and covering factor: closed, gridded aperture, open aperture. It is also confirmed by the presence of absorbing material inside the enclosure which damps the resonances; particularly, when the additional losses are comparable or greater than the intrinsic losses.

The proposed approach that uses HS including FS improves the evaluation of the SE of enclosures in standard IEC 610004-21. HS reduces the MU both at the high and low frequencies. also when the MS is applied only inside the RC.

Compared to the SE in IEEE 299.1, the proposed approach improves the evaluation of the $\mathrm{SE}$ of enclosures at low frequencies.

In any case, the proposed procedure provides more prudent $\mathrm{SE}$ values than the two standards at the low frequencies especially for standard IEEE 299.1.

Note that the proposed procedure could consider both lower values of SE at low frequencies (prudent values from the use of maximum power values) and average values of measured $\mathrm{SE}$ at higher frequencies (from the use of average power values) in order to overcome the greater randomness of the maximum values and reduce the MU over the whole working FR. This is an optimization especially useful for low and moderate measured SE values. We highlight that at the higher frequencies, SE obtained from the ratio between the average values and that obtained from the ratio between the averages of the maximum values are the same in the proposed procedure.

Summing up, it is shown that the procedures for SE of enclosure described in IEC 61000-4-21 [1] and in IEEE 299.1 [13] can be merged in a single enhanced one that takes advantage from the use of FS, the use of maximum (rather than average) values of the measured powers, as well as from the possibility of using a wall-mounted monopole antenna inside the enclosure. For the standard IEC 61000-4-21, such an improvement implies the use of a VNA in the measurement set-up instead of a PM. Note in this regard that the use of the VNA rather than the PM is appealing, since the VNAs are becoming increasingly widespread and their cost is not higher than that of the PMs (considering that use of the PM involves also the use of a radiofrequency source and of a radiofrequency amplifier). According to these considerations, we can say firstly that the proposed approach improves the evaluation of the SE compared to the existing approaches and secondly, it consists of an easy to implement measurement procedure.
Hence, the enhanced, combined, procedure simplifies the standardization by providing a single and better value for SE measurements of enclosures using NRCs. It is an important practical advantage compared to the use of two different standard procedures that provide two different values of SE at the low frequencies.

[1] IEC 61000-4-21, Electromagnetic Compatibility (EMC), Part 4-21: Testing and measurement techniques - Reverberation chamber test methods, International Electrotechnical Commission, Geneva, Switzerland, 2011.

[2] T.A. Loughry, "Frequency stirring: an alternate approach to mechanical mode-stirring for the conduct of electromagnetic susceptibility testing,” Phillips Lab., Rep. PL-TR-91-1036, 1991.

[3] F. Leferink, J.C. Boudenot, and W. Etten, "Experimental results obtained in the intrinsic reverberation chamber," IEEE Int. Symp. Electromagn. Compat., Waschington, D.C., 21-25 August 2000.

[4] U. Carlberg, P.-S. Kildal, A. Wolfang, O. Sotoudeh, C. Orlenius, "Calculated and measured absorption cross sections of lossy objects in reverberation chamber," IEEE Trans. Electromagn. Compat., vol. 46, pp. 146-154, May 2004.

[5] G. Cerri, V.M. Primiani, S. Pennesi, P. Russo "Source stirring mode for reverberation chamber," IEEE Trans. Electromagn. Compat., vol.47, pp. 815-823, November 2005.

[6] U. Carlberg, P. S. Kildal, and J. Carlsson, "Numerical study of position stirring and frequency stirring in a loaded reverberation chamber," IEEE Trans. Electromagn. Compat., vol. 51, pp. 12-17, 2009.

[7] R. Serra, A. Marvin, F. Moglie, W. M. Primiani, A. Cozza, L. Arnaut, Y. Huang, M. Hatfield. M. Klingler and F. Leferink, "Reverberation chambers à la carte: An overview of the different mode-stirring techniques," IEEE Electromagn. Compat. Mag., vol. 6, no. 1, pp. 6378, 2017.

[8] A. Gifuni, L. Bastianelli, M. Migliaccio, F. Moglie, V. Mariani Primiani and G. Gradoni, "On the estimated measurement uncertainty of the insertion loss in a reverberation chamber including frequency stirring," IEEE IEEE Trans. Electromagn. Compat., vol. 61, pp. 1414-1422, Oct. 2019.

[9] S. Lalléchère, C. F. M. Carobbi and L. R. Arnaut, "Review of uncertainty quantification of measurement and computational modeling in EMC Part II: computational uncertainty," IEEE Trans. Electromagn. Compat., vol. 61, pp. 1699-1706, Dec. 2019.

[10] P. Corona, G. Latmiral, and E. Paolini, L. Piccioli, "Use of a reverberating enclosure for measurements of radiated power in the microwave range," IEEE Trans. Electromagn. Compat., vol.18, pp. 54-59, May 1976.

[11] C.L. Holloway, H.A. Haider, R.J. Pirkl, W.F. Yong, D.A. Hill, J. Ladbury, "Reverberation chamber techniques for determining the radiation and total efficiency of antennas," IEEE Trans. Electromagn. Compat., vol.60, pp.1758-1770, April 2012.

[12] C. L. Holloway, D. A. Hill, J. M. Ladbury, P. Wilson, G. Koepke, and J. Coder, "On the use of reverberation chambers to simulate a Rician radio environment for the testing of wireless devices," IEEE Trans. Antennas Propag., vol. 54, no. 11, pp. 3167-3177, Nov. 2006.

[13] IEEE Std 299.1 ${ }^{\mathrm{TM}}-2013$, IEEE Standard Method for Measuring the Effectiveness of Electromagnetic Shielding of Enclosures and Boxes Having all Dimensions between $0.1 \mathrm{~m}$ and $2 \mathrm{~m}$, IEEE, New York, NY 10016-5997, USA, 2013.

[14] Urul, B., Tütüncü, B., \& Helhel, S. "A fast and novel method for determining working volume in the reverberation chamber: Position of Tx antenna affect. Microwave and Optical Technology Letters, vol. 62, pp. 244-250, 2020.

[15] C. L. Holloway, D. A. Hill, M. Sandroni, J. Ladbury, J. Coder, G. Koepke, A. C. Marvin and Y. He, "Use of reverberation chambers to determine the shielding effectiveness of physically small, electrically large enclosures and cavities," IEEE Trans. Electromagn. Compat., vol. 50, no. 4, pp. 770-782, 2008.

[16] A. Gifuni, A. Sorrentino, A. Fanti, G. Ferrara, M. Migliaccio, G. Mazzarella and F. Corona, "On the evaluation of the shielding 
effectiveness of an electrically large enclosure," Adv. Electromagn., vol. 1, no. 1, pp. 84-91, 2012.

[17] I. D. Flintoft, S. J. Bale, A. C. Marvin, M. Ye, J. F. Dawson, C. Wan, M. Zhang, S. L. Parker and M. P. Robinson, "Representative contents design for shielding enclosure qualification from 2 to $20 \mathrm{GHz}$," IEEE Trans. Electromagn. Compat., vol. 60, no. 1, pp. 173-181, 2018.

[18] D. Fedeli, G. Gradoni, V. M. Primiani and F. Moglie, "Accurate analysis of reverberation field penetration into an equipment-level enclosure," IEEE Trans. Electromagn. Compat., vol. 51, no. 2, pp. 170-180, 2009.

[19] Z. Tian, Y. Huang and Q. Xu, "Efficient methods of measuring shielding effectiveness of electrically large enclosures using nested reverberation chambers with only two antennas," IEEE Trans. Electromagn. Compat., vol. 59, no. 6, pp. 1872-1879, 2017.

[20] M. H"oijer and L. Kroon, "Field statistics in nested reverberation chambers," IEEE Trans. Electromagn. Compat., vol. 55, no. 6, pp. 1328-1330, Dec. 2013.

[21] J. Galambos, The Asymptotic Theory of Extreme Order Statistics. New York: Wiley, 1987.

[22] G.J. Freyer, M.O. Hatfield, D.M. Johnson, and M.B. Slocum, "Comparison of measured and theoretical statistical parameters of complex cavities," in IEEE Int. Symp. Electromagn. Compat., Santa Clara, CA, Aug. 1996, pp. 250-253.

[23] T.H. Lehman, and G.J. Freyer, "Characterization of the maximum test level in a reverberation chamber," IEEE 1997 Int. Symp. Electromagn. Compat., Austin, TX, pp. 44-47, August 1997.

[24] N. Wellander, O.Lundén, M.Bäckström "The maximum value distribution in a reverberation chamber," in Proc. IEEE Int. Symp. Electromagn. Compat., Montréal, Canada, August. 13-17, 2001, pp. 751-756.

[25] G.J. Freyer, J.m Lehman, T.H. John M.Ladbury, G.H. Koepke, M.O. Hatfield, "Verification of field applied to an EUT in a reverberation chamber using statistical theory," IEEE 1997 Int. Symp. Electromagn. Compat., Denver, CO, USA, August 1998.

[26] G.Orjubin, "Maximum field inside a reverberation chamber modelled by the generalized extreme value distribution," IEEE Trans. Electromagn. Compat., vol.49, pp. 104-113, February 2007.

[27] A. Gifuni, "Deterministic approach to estimate the upper bound of the electric field in a reverberation chamber," IEEE Trans. Electromagn. Compat., vol. 53, no. 3, pp. 570-578, Aug. 2011.

[28] G. Gradoni and L. R. Arnaut, "Generalized Extreme-Value Distributions of Power Near a Boundary Inside Electromagnetic Reverberation Chambers," in IEEE Trans. Electromagn. Compat., vol. 52, no. 3, pp. 506-515, Aug. 2010.

[29] C. Kasmi, M. Hélier, M. Darces and E. Prouff, "Generalised Pareto distribution for extreme value modelling in electromagnetic compatibility," in Electronics Letters, vol. 49, no. 5, pp. 334-335, 28 Feb. 2013

[30] N. Nourshamsi, J. C. West, C. E. Hager and C. F. Bunting, "Generalized Extreme Value Distributions of Fields in Nested Electromagnetic Cavities," in IEEE Trans. Electromagn. Compat., vol. 61, no. 4, pp. 1337-1344, Aug. 2019.

[31] C. E. Hager, J. D. Rison and G. B. Tait, "Electromagnetic Probabilityof-Effect Assessment Tool for High-Power Susceptibility Testing," in IEEE Trans. Electromagn. Compat., vol. 58, no. 4, pp. 1306-1313, Aug. 2016.

[32] Gilli, M., këllezi, "E. An application of extreme value theory for measuring financial risk," Comput Econ n. 27, pp. 207-228 (2006). https://doi.org/10.1007/s10614-006-9025-7.

[33] H. Penalva, S. Nunes, M. M. Neves, "Extreme value analysis - a brief overview with an application to flow discharge rate data in a hydrometric station in the north of portugal," REVSTAT - Statistical Journ. vol. 14, n. 2, pp. 193-215, April 2016.

[34] B. N. Taylor and C. E. Kuyatt, "Guidelines for Evaluating and Expressing the Uncertainty of NIST Measurement Results," NIST Tech. Note 1297, Sept. 1994".

[35] C. F. M. Carobbi, S. Lalléchère and L. R. Arnaut, "Review of Uncertainty Quantification of Measurement and Computational Modeling in EMC Part I: Measurement Uncertainty," in IEEE Transactions on Electromagnetic Compatibility, vol. 61, no. 6, pp. 1690-1698, Dec. 2019.
[36] M. Hoijer, "Maximum power available to stress onto the critical component in the equipment under test when performing a radiated susceptibility test in the reverberation chamber," in IEEE Transactions on Electromagnetic Compatibility, vol. 48, no. 2, pp. 372-384, May 2006.

[37] F. Corona, "Shielding performance of thin materials definition, modeling, evaluation an enclosure based technique in a reverberating environment," Master's degree thesis in telecommunication engineering, Dipartimento per le Tecnologie, "Parthenope" University, Napoli, Italy, 2010.

[38] A. Gifuni, "Effects of the correction for impedance mismatch on the measurement uncertainty in a reverberation chamber," IEEE Trans. Electromagn.Compat., vol. 57, no. 6, pp. 1724-1727, 2015.

[39] A. Gifuni, G. Gradoni, C. Smartt, S. Greedy, A. M. Villal'on, L. Bastianelli, F. Moglie, V. M. Primiani, S. Perna, and D. Thomas, "Latest developments on the shielding effectiveness measurements of materials and gaskets in reverberation chambers," IET Sci. Meas. Technol., vol. 14, no. 4, pp. 435-445, 2020.

[40] V. Creta, L. Bastianelli, F. Moglie, V. M. Primiani and L. R. Arnaut, "Stirring performance of helically distributed paddles," 2017 IEEE International Symposium on Electromagnetic Compatibility \& Signal/Power Integrity (EMCSI), Washington, DC, 2017, pp. 670674. 